\title{
A VARIATIONAL SOLUTION TO THE PROBLEM OF SCALAR SCATTERING BY A PROLATE SPHEROID*
}

\section{By F. B. Sleator}

1. Introduction. The natural limitation on the variety of problems in scattering and transmission theory which can be solved easily by separation of variables has resulted in a widespread effort to develop alternative approaches. Among these are variational methods, some of which have proved useful in handling certain specific problems, yielding reasonably good approximations at considerably less expense than that entailed by the wave-function and other approaches. However, the integrals involved in these variational expressions are generally of such complexity as to be virtually impossible to handle in more than two dimensions. The spheroid problem, although its geometry is relatively simple and has considerable symmetry, is essentially three-dimensional, and the present solution indicates that in such cases, at least, the integrations required may be feasible, even without restrictions on the wavelength or trial functions.

The procedures employed here in performing these integrations are similar to those appearing elsewhere in the literature in that no pretense to original mathematical rigor is made. Some of the expressions involved are formally divergent, and the order of integration and differentiation is manipulated at will. This situation, of course, appears in many papers on similar problems and has been fully discussed. The results here, at any rate, are physically sensible and can be proved a posteriori to be convergent and unique.

Although the only physical quantity explicitly calculated here is backscattering cross section, it is easy to see how the results could be extended to include certain other quantities of interest. Further refinement of some of the expressions derived might also be profitable under some circumstances. Various possibilities are discussed at the end of the paper.

2. Formulation of the Problem. We assume a rigid prolate spheroid with center at the origin, major axis of length $2 a$ in the $z$-axis, minor axis of length $2 b$, and a plane sound wave approaching in the negative $z$ direction. The following integral equation for the velocity potential at any point exterior to the scatterer can be established:1

$$
\phi(S)=e^{i k z}-(4 \pi)^{-1} \int_{S^{\prime}} \phi\left(S^{\prime}\right)\left(\partial / \partial n^{\prime}\right) G\left(S, S^{\prime}\right) d a^{\prime}
$$

where $S$ is the field point in space, $S^{\prime}$ is the point on the spheroid, $\phi(S)$ is the velocity potential at $S, G\left(S, S^{\prime}\right)$ is the Green's function of free space, $\rho^{-1} \mathrm{e}^{k \hat{\rho}}$

* This paper is based on the writer's Ph.D. thesis investigation. The work was done at the Radiation Laboratory of the University of Michigan and submitted and accepted at New York University.

${ }^{1}$ Baker and Copson, "The Mathematical Theory of Huygens' Principle", Oxford, 1950, p. $26 \mathrm{ff}$. 
( $\rho \equiv$ distance from $S$ to $S^{\prime}$ ), $k=2 \pi / \lambda\left(\lambda=\right.$ wavelength), $\partial / \partial n^{\prime}$ is the derivative in the direction of the exterior normal, and the integration covers the surface.

Application of the boundary condition $\partial \phi / \partial n \mid s^{\prime}=0$ to equation (1) yields

$$
\left.(\partial / \partial n) e^{\imath k z}\right|_{S^{\prime}}=(4 \pi)^{-1} \int_{S^{\prime}} \phi\left(S^{\prime}\right)\left(\partial^{2} / \partial n \partial n^{\prime}\right) G\left(S, S^{\prime}\right) d a^{\prime}
$$

Then it follows that $\phi\left(S^{\prime}\right)$ is the solution of the variational problem $\delta J[\phi]=0$, where

$$
J[\phi]=\frac{\iint_{S} \phi(S)\left(\partial^{2} / \partial n \partial n^{\prime}\right) G\left(S, S^{\prime}\right) \phi\left(S^{\prime}\right) d a d a^{\prime}}{\left[\left.\int_{S} \phi(S)(\partial / \partial n) e^{\imath k z}\right|_{s} d a\right]^{2}} .
$$

It is expedient now to introduce the prolate spheroidal coordinate system $\xi$, $\eta, \phi$, which is related to the rectangular system by the formulas

$$
\begin{aligned}
& x=F \sqrt{\left(\xi^{2}-1\right)\left(1-\eta^{2}\right)} \cos \phi \equiv F \alpha \beta \cos \phi \\
& y=F \sqrt{\left(\xi^{2}-1\right)\left(1-\eta^{2}\right)} \sin \phi \equiv F \alpha \beta \sin \phi \\
& z=F \xi \eta
\end{aligned}
$$

where $F$ is the semi-focal distance, $\alpha \equiv \sqrt{\xi^{2}-1}$, and $\beta \equiv \sqrt{1-\eta^{2}}$. Then if $\sqrt{\xi^{2}-\eta^{2}} \equiv \gamma$ we have

$$
d a=F^{2} \alpha \gamma d \eta d \phi, \quad(\partial / \partial n)=(\alpha / F \gamma)(\partial / \partial \xi) .
$$

The trial function $\phi\left(S^{\prime}\right)$ (for $S^{\prime}$ on the scattering surface) may now be expanded in terms of the Legendre polynomials $P_{\mu}\left(\eta^{\prime}\right)$ which form a complete, orthogonal set over the interval $-1 \leqq \eta^{\prime} \leqq 1$. Thus

$$
\phi\left(S^{\prime}\right)=\sum_{\mu} A_{\mu}\left(\xi^{\prime}\right) P_{\mu}\left(\eta^{\prime}\right)
$$

where the coefficients $A_{\mu}\left(\xi^{\prime}\right)$ are to be determined. Then the variational quantity $J[\phi]$ takes the form

$$
J[\phi]=\frac{\sum_{\mu} \sum_{\nu} A_{\mu} A_{\nu} \iint_{S} P_{\mu}(\eta)\left(\partial^{2} / \partial n \partial n^{\prime}\right) G\left(S, S^{\prime}\right) P_{\nu}\left(\eta^{\prime}\right) d a d a^{\prime}}{\left[\left.\sum_{\nu} A_{\nu} \int_{S} P_{\nu}(\eta)(\partial / \partial n) e^{i k z}\right|_{S} d a\right]^{2}}
$$

or if the integrals in the numerator and denominator are represented by $C_{\mu \nu}$ and $B_{\nu}$ respectively,

$$
J[\phi]=\frac{\sum_{\mu} \sum_{\nu} A_{\mu} A_{\nu} C_{\mu \nu}}{\left[\sum_{\nu} A_{\nu} B_{\nu}\right]^{2}} .
$$

Once values have been obtained for the quantities $C_{\mu \nu}$ and $B_{\nu}$ over a sufficient range of the indices, the problem of finding the back-scattering cross section is relatively trivial. The stationary value of $J[\phi]$ is found by setting the derivative 
of $J$ with respect to each $A_{\mu}$ equal to zero. This operation yields the system of equations

$$
\sum_{\mu=0}^{\infty} A_{\mu}\left[\left(C_{\mu} \nu / B_{\nu}\right)-J_{0} B_{\mu}\right]=0 \text { for all } \nu
$$

where $J_{0}$ is the stationary value of $J[\phi]$. Existence of a solution of this set of homogeneous linear equations in the unknowns $A_{\mu}$ requires that the determinant of the coefficients vanish i.e.,

$$
\left|\left(C_{\mu \nu} / B_{\nu}\right)-J_{0} B_{\mu}\right|=0 \text { or }\left|\left(C_{\mu \nu} / B_{\mu} B_{\nu}\right)-J_{0}\right|=0 .
$$

These are linear equations in $J_{0}$, the solution of which can be written in the form

$$
J_{0}=\left|a_{\mu \nu}\right| / \sum_{\mu} \sum_{v} A_{\mu \nu}
$$

where

$$
a_{\mu \nu} \equiv C_{\mu \nu} / B_{\mu} B_{\nu}, \quad A_{\mu \nu} \equiv \text { cofactor of } a_{\mu \nu} .
$$

Or we can write

$$
J_{0}^{-1}=C^{-1}\left[\left(\left|a_{\mu \nu}+C\right| /\left|a_{\mu \nu}\right|\right)-1\right]
$$

where $C$ is any constant, which is perhaps a more convenient form. Furthermore it develops that $a_{\mu \nu}=0$ for $\mu+\nu$ odd, which means that the coefficients of even and odd index are completely independent, so that if we let $a_{\mu \nu}=\alpha_{\mu \nu}$ for the odd case and $a_{\mu \nu}=\beta_{\mu \nu}$ for the even, we have

$$
J_{0}^{-1}=\frac{\left|\alpha_{\mu \nu}+C_{1}\right|}{C_{1}\left|\alpha_{\mu \nu}\right|}+\frac{\left|\beta_{\mu \nu}+C_{2}\right|}{C_{2}\left|\beta_{\mu \nu}\right|}-\frac{C_{1}+C_{2}}{C_{1} C_{2}}
$$

where $C_{1}$ and $C_{2}$ are arbitrary constants. Once a value is obtained for $J_{0}$, the system (4) can be solved if desired for the coefficients $A_{\mu}$. The back-scattering cross section, however, is given directly by $J_{0}$, which is inversely proportional to the back-scattered amplitude, usually written $f(\pi)$. Specifically, if $\sigma$ is the total scalar back-scattering cross section, then

$$
\sigma=4 \pi\left|J_{0}\right|^{-2} \text {. }
$$

The above solution may also be obtained without resorting to variational language. The method is due to Galerkin and has been shown by Jones ${ }^{2}$ to be exactly equivalent to the variational approach. If the expansion (3) is substituted into the integrand of equation (2), and both sides are then multiplied by $P_{\nu}(\eta)$ and integrated over the spheroid there results the system

$$
\sum_{\mu=0}^{\infty} A_{\mu} C_{\mu \nu}=4 \pi B_{\nu}, \quad \nu=0,1,2 \cdots \infty .
$$

The solution $\left\{A_{\mu}\right\}$ of this system is equivalent (within a normalization constant) to that of the system (4). The value of $J_{0}$ is immediately obtainable from this system by application of the stationary condition

2 Jones, D. S., "A Critique of the Variational Method in Scattering Problems" IRE, Trans. Vol. AP-4, No. 3, (1956), p. 297. 


$$
J_{0} B_{\nu} \sum_{\mu} A_{\mu} B_{\mu}=\sum_{\mu} A_{\mu} C_{\mu \nu} \quad \text { for all } \nu
$$

derived from the definition of $J[\phi]$. Thus

$$
f(\pi)=-J_{0}^{-1}=-(4 \pi)^{-1} \sum_{\mu} A_{\mu} B_{\mu}
$$

and

$$
\sigma=(4 \pi)^{-1}\left|\sum_{\mu} A_{\mu} B_{\mu}\right|^{2} .
$$

This expression is probably more convenient for analytical and computation purposes than those derived above, and is used in the developments which follow.

Evaluation of the integrals $B_{\nu}$ and $C_{\mu \nu}$ is described in Appendix B. The former is trivial, once the proper substitutions are made, but the latter offers considerable difficulty. Use of the triple integral expression for the Green's function results in a seven-fold integration to be performed. Six of the integrations have been carried out, but the seventh appears to be too complicated for treatment by the usual analytical techniques, and the actual evaluation was done graphically. Final forms of the coefficients are the following:

$$
\begin{gathered}
B_{\nu}=\left.\int_{S} P_{\nu}(\eta) \frac{\partial e^{i k z}}{\partial n}\right|_{s} d a=4 \pi F^{2} \alpha^{2} i^{\nu} k \frac{d \psi_{\nu}(k a)}{d(k a)} \\
C_{\mu \nu}=\iint_{S} P_{\mu}(\eta) \frac{\partial^{2} G\left(S, S^{\prime}\right)}{\partial n \partial n^{\prime}} P_{\nu}\left(\eta^{\prime}\right) d a d a^{\prime} \\
=-8 \pi^{2} F^{4} \xi^{2} \alpha^{3} k^{3} i^{\mu+3 v+1} \int_{0}^{\pi} \Gamma_{\mu}(\xi, k, \psi) \Delta_{\nu}(\xi, k, \psi)\left(\xi^{2}-\cos ^{2} \psi\right)^{-1} \sin \psi d \psi
\end{gathered}
$$

for $\mu \leqq \nu$, where

$$
\Gamma_{\mu}(\xi, k, \psi)
$$

$$
\equiv \mu \rho^{-1}\left[\cos \psi P_{\mu-1}(\cos \psi)-\xi^{2} P_{\mu}(\cos \psi)\right] \zeta_{\mu}(\rho)+\left(\xi^{2}-\cos ^{2} \psi\right) P_{\mu}(\cos \psi) \zeta_{\mu+1}(\rho)
$$

$\Delta_{\nu}(\xi, k, \psi)$

$$
\equiv \nu \rho^{-1}\left[\cos \psi P_{\nu-1}(\cos \psi)-\xi^{2} P_{\nu}(\cos \psi)\right] \psi_{\nu}(\rho)+\left(\xi^{2}-\cos ^{2} \psi\right) P_{\nu}(\cos \psi) \psi_{\nu+1}(\rho)
$$

and $\rho \equiv k F \xi \alpha\left(\xi^{2}-\cos ^{2} \psi\right)^{-\frac{1}{2}}$. Furthermore $C_{\mu \nu}=0$ for $\mu+\nu$ odd, since the integrand is then odd about $\psi=\pi / 2$, and $C_{\mu \nu}$ must be symmetrical in $\mu$ and $\nu$, so that for $\mu>\nu$ the subscripts on the factors $\Gamma_{\mu}$ and $\Delta_{\nu}$ must be interchanged.

3. Verification of Results. The most obvious check on the validity of the solution obtained here is afforded by a comparison with known solutions for the extreme values of wavelength and eccentricity. Considering the latter, it is apparent that as the spheroid becomes infinitely thin, the cross section should vanish for any finite value of $k a$, and the forms obtained actually vanish, since $\alpha$ goes to zero. More interesting is the opposite extreme, where the eccentricity vanishes, i.e. the spheroid becomes a sphere. This transformation is accomplished by letting $\xi \rightarrow x$ and $F \rightarrow 0$ in such a way that $\xi F \rightarrow a$, the radius of the sphere. 
Applying this to the expressions (8) and (9), we find that

$$
\begin{aligned}
& B_{\nu} \rightarrow 4 \pi a^{2} i^{\mu} k \frac{d \psi_{\nu}(k a)}{d(k a)} \\
& C_{\mu \nu} \rightarrow \begin{cases}0 & \text { for } \mu \neq \nu \\
-\left(\frac{16 \pi^{2} i k^{3} a^{4}}{(2 \mu+1)}\right)\left(\frac{d \zeta_{\mu}(k a)}{d(k a)}\right)\left(\frac{d \psi_{\mu}(k a)}{d(k a)}\right) & \text { for } \mu=\nu\end{cases}
\end{aligned}
$$

Moreover the system (5) reduces to

$$
A_{\mu} C_{\mu \mu}=4 \pi B_{\mu} \text { for all } \mu,
$$

and thus

$$
f(\pi)=-\sum_{\mu} \frac{B \mu^{2}}{C_{\mu \mu}}=\frac{i}{k} \sum_{\mu}(-1)^{\mu}(2 \mu+1) \frac{d \psi_{\mu}(k a)}{d(k a)} / \frac{d \xi_{\mu}(k a)}{d(k a)} .
$$

This is identical to the classical solution for the sphere, as given for example by Sommerfeld, ${ }^{3}$ restricted to the case of back-scattering in the far zone.

The essential nature of the series used here indicates that the approximation improves as the wavelength increases, and accordingly the variational solution should agree with the Rayleigh result in the region of large wavelength. That it actually does agree can be shown in the following manner.

If the coefficients $A_{\mu}, B_{\nu}$, and $C_{\mu \nu}$ are expanded as power series in $k$, and these series are substituted in the system (5) then equating coefficients of like powers of $k$ yields a linear system of equations in the coefficients of these series which is essentially triangular. Thus if the coefficients in the expansions of the $B_{\nu}$ and $C_{\mu \nu}$ are known, the system can be solved for those of the $A_{\mu}$ to any degree desired. The former coefficients are easily obtained by substituting power series expansions of the Bessel and Hankel functions into the expressions (8) and (9). The resulting coefficients of the $A_{\mu}$ can be used along with those of the $B_{\nu}$ in the series (6) for the scattered amplitude, and the terms of order less than or equal to $k^{2}$ should give the Rayleigh result. In the present case if we set

$$
A_{\nu}=\sum_{j} a_{j}^{\nu} k^{j}, \quad B_{\nu}=\sum_{j} b_{j}^{\nu} k^{j}, \quad C_{\mu \nu}=\sum_{j} c_{j}^{\mu \nu} k^{|\nu-\mu|+j}
$$

we find that the only non-vanishing coefficients of the $B_{\nu}$ and $C_{\mu \nu}$ required are

$$
b_{2}^{0}=-(4 / 3) \pi F^{3} \alpha^{2} \xi, \quad b_{1}^{1}=(4 / 3) \pi i F^{2} \alpha^{2}
$$

and

$$
c_{2}^{00}=-(16 / 2) \pi^{2} F^{3} \alpha^{2} \xi, \quad c_{0}^{11}=(16 / 3) \pi^{2} F \alpha^{2}\left\{\xi-\frac{1}{2} \alpha^{2} \log [(\xi+1) /(\xi-1)]\right\}
$$

The triangular system of equations for the $a_{j}^{\mu}$ reduces to

$$
c_{2}^{00} a_{0}^{0}=4 \pi b_{2}^{0}, \quad c_{0}^{11} a_{1}^{1}=4 \pi b_{1}^{1}
$$

and thus

$$
a_{0}^{0}=1, \quad a_{1}^{1}=i F\left\{\xi-\frac{1}{2} \alpha^{2} \log [(\xi+1) /(\xi-1)]\right\}^{-1} .
$$

${ }^{3}$ Sommerfeld, A., "Partial Differential Equations in Physics", Academic Press. (1949), p. 164. 
When the above values are substituted in the expression (6) for the scattered amplitude, the result is

$$
\begin{aligned}
& f(\pi) \approx-\left(k^{2} / 4 \pi\right)\left[a_{0}^{0} b_{2}^{0}+a_{1}^{1} b_{1}^{1}\right] \\
& \approx\left(k^{2} / 3\right) F^{3} \alpha^{2}\left\{\xi^{2}+1-\frac{1}{2} \xi \alpha^{2} \log [(\xi+1) /(\xi-1)]\right\} \\
& \cdot\left\{\xi-\frac{1}{2} \alpha^{2} \log [(\xi+1) /(\xi-1)]\right\}^{-1}
\end{aligned}
$$

which is identical to the Rayleigh result for the corresponding case.

This procedure is extended in Appendix A to obtain the second and third (non-vanishing) coefficients in the series, and the resulting approximation is compared with the exact solution for a particular spheroid.

In the limit of small wavelength the analysis is more difficult. It can be shown, however, that the situation is similar to that in the sphere solution, in that the number of terms in the series (6) required is of the order of $k a$. Use of asymptotic expressions for the Bessel functions appearing in (9) permits a stationary phase approximation to the integral, and it then develops that each term in the series (6) with $\mu \ll k a$ is of order $1 / k a$, so that a reasonable approximation to the scattered field must include at least $k a$ terms.

The principal mathematical question remaining is that of convergence of the series for the scattered amplitude. The analysis of this problem is necessarily tedious, but it can be shown, using even the roughest of estimates and bounds for the significant quantities, that the solution does indeed converge for a certain range of $k a$, and it is perhaps reasonable to assume that it converges for any finite value. Some indication of this is given by the rate at which the Bessel functions appearing in the expressions for the $B_{\nu}$ and $C_{\mu \nu}$ die out with increasing index. This insures that the coefficients $B_{\nu}$ and $C_{\mu \nu}$ satisfy certain conditions which are sufficient to guarantee the existence of a bounded solution to the linear system (5), and the boundedness of the $A_{\nu}$ is sufficient for the convergence of the series $\sum_{\mu} A_{\mu} B_{\mu}$.

4. Numerical Results. On the basis of the forms developed in the preceding sections a value was computed for the nose-on back-scattering cross section of a particular spheroid at a single wavelength. In order to obtain a comparison with the exact solution, parameter values were chosen for which the latter had previously been determined. ${ }^{4}$ The axis ratio of the spheroid was taken as $10: 1$, and the wavelength ratio $k a$ was given the value 1.40 , which is very near the location of the first maximum in the curve of cross section vs. $k a$. The integrals in equation (9) were evaluated by means of Simpson's rule, using intervals of $.5^{\circ}$ in the range $0 \leqq \psi<10^{\circ}$ and $2.5^{\circ}$ in the range $10 \leqq \psi \leqq 90^{\circ}$. (The smaller intervals near the origin were necessary because with the value of $\xi$ very near unity, the denominator of the integrand is small in this region and the value of the integrand in general rises quite sharply. This effect would be less pronounced for a fatter spheroid). The linear system (5) was then solved, under the usual

4 Siegel, K. M., et al., "Theoretical and Numerical Determination of the Radar Cross Section of a Prolate Spheroid", IRE Trans. Vol. AP. 4, No. 3 (1956), p. 266. 
TABLE I

\begin{tabular}{|l|l|l|l||c|c|}
\hline & & \multicolumn{2}{|c|}{$\mathrm{C}_{\mu \nu}\left[16 \pi^{2} \mathrm{k}^{2}{ }^{3} \alpha^{2} / \xi^{2}\right]^{-1}$} & & \\
\hline & $\nu$ & $\mathrm{Re}$ & $\mathrm{B}_{\nu} / \mathrm{a}$ \\
\hline 0 & 0 & $2.68085 \cdot 10^{-3}$ & $-3.40193 \cdot 10^{-1}$ & 0 & $-5.33925 \cdot 10^{-3}$ \\
\hline 0 & 2 & $9.36340 \cdot 10^{-5}$ & $-6.38906 \cdot 10^{-2}$ & 1 & $2.22699 \cdot 10^{-3}$ \\
\hline 0 & 4 & $1.58590 \cdot 10^{-6}$ & $-1.37306 \cdot 10^{-4}$ & 2 & $-1.93905 \cdot 10^{-3}$ \\
\hline 2 & 2 & $7.13970 \cdot 10^{-5}$ & $2.46674 \cdot 10^{-1}$ & 3 & $-6.50311 \cdot 10^{-4} \mathrm{i}$ \\
\hline 2 & 4 & $1.85214 \cdot 10^{-6}$ & $-1.60819 \cdot 10^{-2}$ & 4 & $1.41940 \cdot 10^{-4}$ \\
\hline 4 & 4 & $1.11642 \cdot 10^{-7}$ & $4.74239 \cdot 10^{-1}$ & & \\
\hline 1 & 1 & $1.45043 \cdot 10^{-4}$ & $1.01764 \cdot 10^{-1}$ & & \\
\hline 1 & 3 & $4.66476 \cdot 10^{-6}$ & $-2.57312 \cdot 10^{-2}$ & & \\
\hline 3 & 3 & $3.53351 \cdot 10^{-6}$ & $3.69719 \cdot 10^{-1}$ & & \\
\hline
\end{tabular}

truncation assumptions, as an $N \times N$ system, and the order $N$ was given several values in order to obtain some indication of the convergence rate. Values of the scattering cross section $\sigma$ were computed from equation (7) and divided by the geometric optics result $\pi b^{4} / a^{2}$ for convenience in comparing with the known solution. The value of the latter at the point in question, to five significant figures, is

$$
\frac{\sigma}{\pi b^{4} / a^{2}}=1.1022
$$

The following table contains the values computed from the variational result, listed as a function of the order $N$ of the linear system.

$$
\begin{array}{rlcccc}
N & =1 & 2 & 3 & 4 & 5 \\
\frac{\sigma}{\pi b^{4} / a^{2}} & =.7337 & 1.833 & 1.025 & 1.110 & 1.105
\end{array}
$$

The fifth order result is seen to agree with the exact answer within about $.3 \%$ at this point.

Some of the intermediate quantities used in obtaining these figures may also be of interest and are tabulated here for the sake of completeness. The coefficients $C_{\mu \nu}$ and $B_{\nu}$, with certain common factors removed for convenience, are listed in Table $I$.

Table II contains the values of $A_{\mu}$ which result as the solution to the fifth order linear system. Substitution of these quantities in equation (3) gives the potential distribution on the scattering surface.

Several possibilities might be considered in an attempt to improve the accuracy of the variational result. The discrepancy between this and the exact 
TABLE II

\begin{tabular}{|c|c|c|}
\hline$\mu$ & $\operatorname{Re} A_{\mu}$ & $\operatorname{Im} A_{\mu}$ \\
\hline 0 & $6.92536 \cdot 10^{-1}$ & $-5.44415 \cdot 10^{-3}$ \\
\hline 1 & $-1.69158 \cdot 10^{-3}$ & 1.15989 \\
\hline 2 & $-5.82917 \cdot 10^{-1}$ & $9.76788 \cdot 10^{-4}$ \\
\hline 3 & $1.31725 \cdot 10^{-4}$ & $-1.70612 \cdot 10^{-1}$ \\
\hline 4 & $3.52111 \cdot 10^{-2}$ & $-3.00564 \cdot 10^{-5}$ \\
\hline
\end{tabular}

answer must be attributable primarily to three factors: 1) round-off error in the numerical quantities, 2) approximations inherent in numerical integration, and 3) truncation of the series. The first of these appears to be most significant in the present case. The accuracy employed throughout the computations was six decimal places, and a fifth-order answer was also computed using intervals of twice the above specified lengths in the integration process. This yielded a value of 1.1025 for the quantity in question, which is somewhat more accurate than the value obtained with the shorter intervals, indicating that the point of diminishing returns may already have been passed in the direction of refining the integration intervals. Regarding the third factor, it seems that the successive orders of approximation form an oscillating sequence, and since the fifth-order answer is between the fourth and the correct value, it is to be expected that a sixth-order result, employing the same decimal accuracy, might be worse than the fifth.

The problem of maintaining greater accuracy in the numerical quantities might be troublesome for hand computation, due to limitations in the accuracy of available tables of the special functions and the necessity for complicated interpolation methods; however for a large scale computing machine it should not prove difficult.

5. Conclusions. In view of the results outlined above there can be little doubt that the solution obtained here is actually valid. Its utility, however, is perhaps harder to establish. In its present form, the variational solution seems to be comparable to the wave-function solution in accuracy and economy of computation. The former has perhaps a slight edge in cases where the existing tables of spheroidal coefficients do not apply, but the graphical or numerical evaluation of the remaining integrals is tedious in any case, and the accuracy required is considerable. Some thought has been given to means of carrying out the final integrations analytically. Various expansions and partial integrations have been tried but without any noteworthy success. The number of terms required at moderate values of $\mu$ and $\nu$ is in general prohibitive.

For values of $k a$ up to about 1.0 (for the 10:1 spheroid) an excellent approximation to the cross section is given by the three-term power series described in Appendix A, which is of course much more convenient than either the varia- 
tional or wave-function result. It appears that one more term in this series might give a good approximation to the first maximum in the curve of cross section vs. $k a$, but the algebra involved in obtaining this term is rather formidable, and since the series can be expected to diverge in the near vicinity of this maximum, there is some doubt about the value of the result. The accuracy of the threeterm approximation for other spheroids is also open to question. Computations done elsewhere ${ }^{5}$ for the electromagnetic case show that in the range $k a<1$ a two-term approximation for the cross section falls well above the exact curve for the sphere and below it for a 10:1 spheroid. The accuracy thus depends rather significantly on the eccentricity, and it seems probable that the particular case computed here was nearly optimum in this respect.

Although the back-scattering cross section is the only physical quantity calculated explicitly in the foregoing, it is clear that certain other information could be obtained fairly easily. The values of the $A_{\nu}$ listed previously yield the potential layer on the scattering surface at once. Also, if the integral in the original integral equation (1) can be resolved after substituting the expansion (3) for the trial function, the result will give a five-term approximation for the scattered field at any point in space. It should be pointed out, however, that the single integration over the spheroid may offer more difficulty than the double one actually performed, since it represents a more general expression.

One more unanswered mathematical question arises in connection with the bearing of the present solution on the utility of variational methods in general. A result was obtained here largely by virtue of the separability of the integrations appearing in the expression (13) for the coefficients $C_{\mu \nu}$. One is led to suspect the existence of an intimate connection between this and the separability of the wave equation in the coordinate system being used, and if this is so, the hope of employing similar techniques to obtain variational solutions to nonseparable three-dimensional problems is seriously diminished.

Appendix A: Power Series Solution. The development in Section III indicates that the variational forms may be used in deriving a power series representation for the scattered field. The procedure and results in the case of the circular aperture problem have been discussed by Magnus. ${ }^{6}$ For the case of an electromagnetic wave striking an ellipsoid, the first two non-vanishing coefficients have been derived by Stevenson ${ }^{7}$ without reference to any variational expressions. Whether the latter offer any material advantage in deriving these and subsequent coefficients in the scalar problem is not immediately clear. At any rate they have been utilized to obtain the second and third coefficients for the prolate spheroid, and the results are given here. The derivation proceeds along the

\footnotetext{
${ }^{5}$ Mathur, P., and Mueller, E., "Radar Back-Scattering Cross Sections for Non-spherical Targets", IRE-Trans, Vol. AP-4, No. 1, Jan. 1956.

'Magnus, W., "Infinite Matrices Associated with Diffraction by an Aperture", Quarterly of Applied Math. 11, (1953), pp. 77-86.

${ }^{7}$ Stevenson, A. F., "Electromagnetic Scattering by an Ellipsoid in the Third Approximation", Jour. Applied Physics, 24, Sept. 1953.
} 
lines described in Section III. It is straightforward but tedious, and details will be omitted here.

We are concerned with an expression for the scattered field $f(\pi)$ of the form

$$
f(\pi)=\sum_{n} R_{n} k^{n}
$$

It was shown earlier that the coefficients of index 0 and 1 vanish identically. Stevenson has shown that in the electromagnetic case at least the coefficient $R_{3}$ also vanishes. It develops that this is also true in the scalar problem, and that here $R_{5}$ vanishes as well, though $R_{7}$ apparently does not. We will limit ourselves to determination of expressions for $R_{4}$ and $R_{6}$.

When the power series expansions of the quantities $A_{\mu}, B_{\nu}$, and $C_{\mu \nu}$ are substituted into the linear system (5) and the expression (6) for the scattered field, it is easily shown that the $n^{\text {th }}$ coefficient in the series (11) can be written as an inner product

$$
R_{n}=-\frac{1}{2} b^{2} a^{-\frac{1}{3}} \mathbf{A}_{n} \cdot \mathbf{B}_{n}
$$

where $\mathbf{A}_{n}$ and $\mathbf{B}_{n}$ are vectors whose components are proportional to certain coefficients in the power series expansions of $A_{v}$ and $B_{v}$ respectively, and that furthermore

$$
\mathbf{A}_{n}=-2 a^{-\frac{5}{2}} C_{n}^{-1} \mathbf{B}_{n}
$$

where $C_{n}^{-1}$ is the inverse of a matrix $C_{n}$ whose elements are proportional to certain coefficients in the series for $C_{\mu \nu}$. Combining (12) and (13) we can write $R_{n}$ as a quadratic form

$$
R_{n}=(b / a)^{2} \mathbf{B}_{n} \cdot C_{n}^{-1} \mathbf{B}_{n} .
$$

It can also be shown that the dimensions of $\mathbf{A}_{n}$ and $\mathbf{B}_{n}$ should in general be $(1 / 4)(n+2)^{2}$ for $n$ even, or $(1 / 4)(n+1)(n+3)$ for $n$ odd, though in the present problem a number of the components vanish, so that the dimension is actually less than the specified value in each case considered. Moreover, because of the vanishing of $C_{\mu \nu}$ for $\mu+\nu$ odd, it develops at once that the matrix $C_{n}$ is the direct sum of two submatrices, one deriving from the even values of $\mu$ and $\nu$ and the other from the odd, so that the transformation is considerably simplified. For the computation of $R_{4}$ and $R_{6}$ the essential forms are as follows: The quantities $B_{\nu}$ and $C_{\mu \nu}$ are expanded in the power series

$$
\begin{aligned}
B_{\nu} & =2 \pi b^{2} a^{-\frac{1}{2}} \sum_{r=0}^{\infty} b_{r}^{\nu} k^{r} \\
C_{\mu \nu} & ==4 \pi^{2} b^{2} a \sum_{S=0}^{\infty} c_{S}^{\mu \nu} k^{|\nu-\mu|+s} .
\end{aligned}
$$

(This notation differs slightly from that in Section III in that for the sake of economy we have removed common factors from the coefficients $b_{r}^{\nu}$ and $c_{s}^{\mu \nu}$.) The vector $B_{4}$ may then be written

$$
\mathbf{B}_{4} \equiv\left(b_{4}^{0}, b_{2}^{0}, b_{2}^{2}, b_{3}^{1}, b_{1}^{1}\right)
$$

and the corresponding matrix $C_{4}$ is 
TABLE III

\begin{tabular}{|c|c|c|c|c|c|c|}
\hline \multicolumn{7}{|c|}{ Values of $b_{r}$} \\
\hline & 1 & 2 & 3 & 4 & 5 & 6 \\
\hline 0 & & $-2 a / 3$ & & $a^{3} / 15$ & & $-\mathrm{a}^{5} / 420$ \\
\hline 1 & $2 i / 3$ & & $\mathrm{ia}^{2} / 5$ & & $\mathrm{ia}^{4} / 84$ & \\
\hline 2 & & $-4 a / 15$ & & $4 a^{3} / 105$ & & \\
\hline 3 & & & $\mathrm{ia}^{2} / 35$ & & & \\
\hline
\end{tabular}

$$
C_{4}=\left[\begin{array}{ccc}
c_{4}^{00} & c_{2}^{00} & c_{0}^{02} \\
c_{2}^{00} & 0 & 0 \\
c_{0}^{02} & 0 & c_{0}^{22}
\end{array}\right]+\left[\begin{array}{cc}
c_{2}^{11} & c_{0}^{11} \\
c_{0}^{11} & 0
\end{array}\right]
$$

Similarly we can write

$$
\mathbf{B}_{6} \equiv\left(b_{2}^{0}, b_{2}^{2}, b_{4}^{0}, b_{4}^{2}, b_{6}^{0}, b_{1}^{1}, b_{3}^{\mathbf{1}}, b_{3}^{3}, b_{5}^{\mathbf{1}}\right)
$$

and

$$
C_{6}=\left[\begin{array}{ccccc}
0 & 0 & 0 & 0 & c_{2}^{00} \\
0 & 0 & 0 & c_{0}^{22} & c_{0}^{02} \\
0 & 0 & c_{2}^{00} & c_{0}^{02} & c_{4}^{00} \\
0 & c_{0}^{22} & c_{0}^{02} & c_{2}^{22} & c_{2}^{02} \\
c_{2}^{00} & c_{0}^{02} & c_{4}^{00} & c_{2}^{02} & c_{6}^{00}
\end{array}\right]+\left[\begin{array}{cccc}
0 & 0 & 0 & c_{0}^{11} \\
0 & c_{0}^{11} & 0 & c_{2}^{11} \\
0 & 0 & c_{0}^{33} & c_{0}^{13} \\
c_{0}^{11} & c_{2}^{11} & c_{0}^{13} & c_{4}^{11}
\end{array}\right]
$$

The values of $b_{r}^{\nu}$ and $c_{s}^{\mu \nu}$ are obtainable from the forms appearing in Section II. The general expressions are given in Tables III and IV.

The matrices $C_{4}$ and $C_{6}$ are easily inverted, and the above expressions along with equation (10) may be used to obtain the first three coefficients in the power series solution for an arbitrary spheroid. For the 10:1 spheroid the values of $\xi$ and $\alpha$ are 1.0050 and .1005 respectively, and substitution of these in the above gives the following values of the $R_{n}$ :

$$
R_{2}=6.736 a^{3} \cdot 10^{-3}, R_{4}=2.838 a^{5} \cdot 10^{-3}, R_{6}=2.922 a^{7} \cdot 10^{-4},
$$


TABLE IV

\begin{tabular}{|c|c|c|c|}
\hline$\mu$ & $\nu$ & $\mathbf{s}$ & Values of $c_{s}^{\mu \nu}$ \\
\hline 0 & 0 & 2 & $4 / 3$ \\
\hline 0 & 0 & 4 & $\frac{4 \mathrm{a}^{2} \alpha^{2}}{15 \xi} \log \frac{\xi+1}{\xi-1}$ \\
\hline 0 & 0 & 6 & $\frac{-2 a^{4} \alpha^{2}}{35 \xi^{3}}\left[2 \xi+\alpha^{2} \log \frac{\xi+1}{\xi-1}\right]$ \\
\hline 0 & 2 & 0 & $\frac{2}{15}\left[-2\left(3 \xi^{2}-2\right)+3 \xi \alpha^{2} \log \frac{\xi+1}{\xi-1}\right]$ \\
\hline 0 & 2 & 2 & $\frac{4 a^{2} \alpha^{2}}{105 \xi}\left[6 \xi-\left(3 \xi^{2}-1\right) \log \frac{\xi+1}{\xi-1}\right]$ \\
\hline 2 & 2 & 0 & $\frac{6 \xi^{2}}{5 \mathbf{a}^{2}}\left[-2\left(3 \xi^{2}-2\right)+3 \xi \alpha^{2} \log \frac{\xi+1}{\xi-1}\right]$ \\
\hline 2 & 2 & 2 & $\frac{4}{105}\left[\left(54 \xi^{4}-60 \xi^{2}+11\right)+3 \xi \alpha^{2}\left(9 \xi^{2}-4\right) \log \frac{\xi+1}{\xi-1}\right]$ \\
\hline 1 & 1 & 0 & $\frac{2 \xi}{3 \mathbf{a}^{2}}\left[-2 \xi+\alpha^{2} \log \frac{\xi+1}{\xi-1}\right]$ \\
\hline 1 & 1 & 2 & $\frac{2}{15 \xi}\left[2 \xi\left(3 \xi^{2}-2\right)-\left(3 \xi^{2}-1\right) \alpha^{2} \log \frac{\xi+1}{\xi-1}\right]$ \\
\hline 1 & 1 & 4 & $\frac{a^{2} \alpha^{2}}{210 \xi^{3}}\left[-2 \xi\left(23 \xi^{2}+3\right)+\left(23 \xi^{4}-18 \xi^{2}+3\right) \log \frac{\xi+1}{\xi-1}\right]$ \\
\hline 1 & 3 & 0 & $\frac{1}{70 \xi}\left[2 \xi\left(45 \xi^{4}-48 \xi^{2}+7\right)-\left(15 \xi^{2}-1\right)\left(3 \xi^{2}-1\right) \alpha^{2} \log \frac{\xi+1}{\xi-1}\right]$ \\
\hline 3 & 3 & 0 & $\frac{3 \xi\left(5 \xi^{2}-1\right)}{14 a^{2}}\left[-2 \xi\left(5 \xi^{2}-13\right)+3\left(5 \xi^{2}-1\right) \alpha^{2} \log \frac{\xi+1}{\xi-1}\right]$ \\
\hline
\end{tabular}

For comparison with the exact solution it is convenient to obtain the corresponding series representation of the quantity $\sigma /\left(\pi b 4 / a^{2}\right)=\sum_{n} S_{n}(k a)^{n}$. We obtain as the first three non-vanishing coefficients

$$
\begin{aligned}
& S_{4}=4(a / b)^{4}\left(R_{2} / a^{3}\right)^{2}=1.8148 \\
& S_{6}=8(a / b)^{4}\left(R_{2} / a^{3}\right)\left(R_{4} / a^{5}\right)=1.5296 \\
& S_{8}=4(a / b)^{4}\left[\left(R_{4} / a^{5}\right)^{2}+\left(2 R_{2} / a^{3}\right)\left(R_{6} / a^{7}\right)\right]=.4796
\end{aligned}
$$

The accompanying graph shows how the exact curve is approximated by the power series representations. It appears that at this value of the eccentricity 


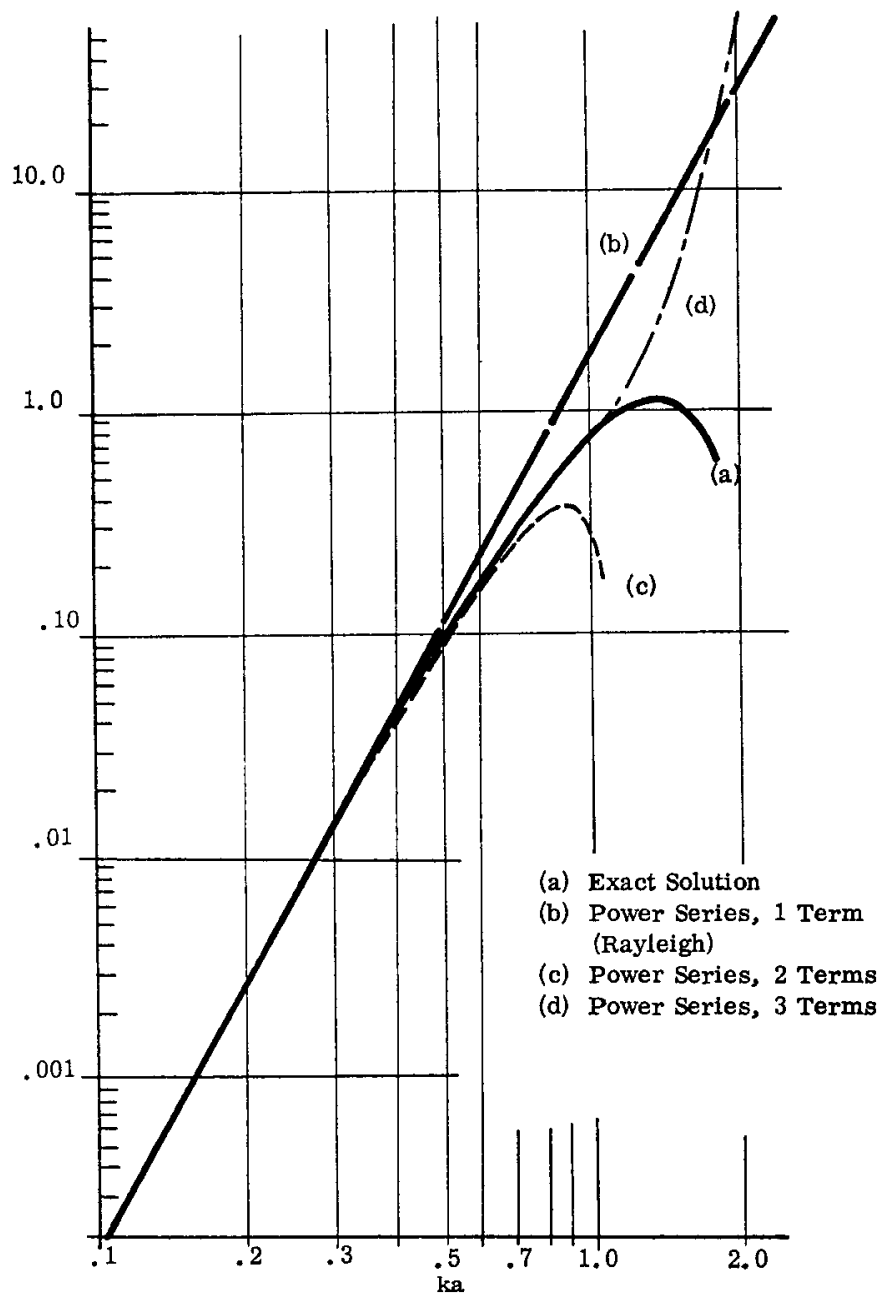

BACK-SCATTERING CROSS SECTION

$$
\frac{\sigma}{\pi b^{4} / a^{2}}
$$

23

FIG. 1

the three-term expression gives an excellent approximation to the correct curve out to about $k a=1.0$.

Appendix B: Evaluation of the Integrals. The integral

$$
\left.B_{\nu} \equiv \int_{S} P_{\nu}(\eta)(\partial / \partial n) e^{i k z}\right|_{s} d a
$$

can be evaluated easily as a special case of the following integral of Gegenbauer (a general form is listed since it is required in the evaluation of the $C_{\mu \nu}$ ): 


$$
\int_{-1}^{1} e^{i z \eta \cos \psi} J_{m}\left(z \sqrt{1-\eta^{2}} \sin \psi\right) P_{n}^{m}(\eta) d \eta=i^{n-m} \sqrt{2 \pi / z} P_{n}^{m}(\cos \psi) J_{n+\xi}(z)
$$

for $0 \leqq \psi \leqq \pi$. We have

$$
\begin{aligned}
B_{\nu} & =\left.F \alpha^{2} \int_{0}^{2 \pi} \int_{-1}^{1} P_{\nu}(\eta)(\partial / \partial \xi) e^{i k F \xi \eta}\right|_{s} d \eta d \phi=2 \pi i F^{2} \alpha^{2} k \int_{-1}^{1} P_{\nu}(\eta) e^{i k a \eta} \eta d \eta \\
& =\frac{2 \pi i F^{2} \alpha^{2} k}{(2 \nu+1)}\left\{(\nu+1) \int_{-1}^{1} P_{\nu+1}(\eta) e^{i k a \eta} d \eta+\nu \int_{-1}^{1} P_{\nu-1}(\eta) e^{i k a \eta} d \eta\right\} .
\end{aligned}
$$

These integrals correspond to the form above with $m=0, \psi=0$, and $z=k a$. Thus

$$
B_{\nu}=\frac{\left(2 \pi F^{\prime}\right)^{1} \alpha^{\nu} \alpha^{2} \sqrt{k}}{(2 \nu+1) \sqrt{\xi}}\left[\nu J_{\nu-\frac{1}{\xi}}(k a)-(\nu+1) J_{\nu+\xi}(k a)\right]
$$

or in terms of the spherical Bessel functions

$$
B_{\nu}=4 \pi F^{2} \alpha^{2} i^{\eta} k \frac{d \psi_{v}(k a)}{d(k a)}
$$

Details of the evaluation of the $C_{\mu \nu}$ integrals are too extensive to include here. There follows an outline of the procedure showing the essential steps in the derivation of the expression (9).

To begin with it is advantageous to use the Fourier integral representation of the Green's function of free space

$$
G\left(S, S^{\prime}\right) \equiv \frac{e^{\imath k \rho}}{\rho}=\frac{1}{2 \pi^{2}} \iiint_{-\infty}^{\infty} \frac{e^{i \mathbf{K} \cdot \boldsymbol{\ell}}}{\mathbf{K}^{2}-k^{2}} d \mathbf{K}
$$

where

$$
\mathbf{K}=\left(K_{x}, K_{y}, K_{z}\right), d \mathbf{K}=d \mathbf{K}_{x} d \mathbf{K}_{y} d \mathbf{K}_{z}, \mathbf{K}^{2}=\mathbf{K K}=K_{x}^{2}+K_{y}^{2}+K_{z}^{2}
$$

and

$$
\mathbf{o}=\left(x-x^{\prime}, y-y^{\prime}, z-z^{\prime}\right) .
$$

This can be formally differentiated twice in the directions $n$ and $n^{\prime}$ to give

$$
\begin{aligned}
\frac{\partial^{2} G}{\partial n \partial n^{\prime}}=\frac{\alpha \alpha^{\prime}}{2 \pi^{2} \gamma \gamma^{\prime}} \iiint_{-\infty}^{\infty} \frac{e^{i \mathrm{~K} \cdot \boldsymbol{\theta}}}{\mathrm{K}^{2}-k^{2}}\left(K_{x} \frac{\beta}{\alpha} \xi \cos \phi+K_{y} \frac{\beta}{\alpha} \xi \sin \phi+K_{z} \eta\right) \\
\cdot\left(K_{x} \frac{\beta^{\prime}}{\alpha^{\prime}} \xi^{\prime} \cos \phi^{\prime}+K_{y} \frac{\beta^{\prime}}{\alpha^{\prime}} \xi^{\prime} \sin \phi^{\prime}+K_{z} \eta^{\prime}\right) d \mathbf{K} .
\end{aligned}
$$

This quantity should properly be regarded as a limit as $\xi^{\prime} \rightarrow \xi$, i.e. the distinction between these surfaces must be preserved until after the critical integrations are performed, in order to avoid difficulty with the singularity at $S=S^{\prime}$. Then with some rearrangement of the integrals, we can write 


$$
\begin{aligned}
C_{u \nu}=\frac{F^{4} \alpha^{4}}{2 \pi^{2}} \lim _{\xi^{\prime} \rightarrow \xi} \iiint_{-\infty}^{\infty} \frac{d \mathbf{K}}{\mathbf{K}^{2}-k^{2}} \iint_{-1}^{1} \iint_{0}^{2 \pi} e^{i \mathbf{K} \cdot \boldsymbol{\theta}} P_{\mu}(\eta) P_{y}\left(\eta^{\prime}\right) \\
\cdot\left(K_{x} \frac{\beta}{\alpha} \xi \cos \phi+K_{y} \frac{\beta}{\alpha} \xi \sin \phi+K_{z} \eta\right) \\
\\
\cdot\left(K_{x} \frac{\beta^{\prime}}{\alpha^{\prime}} \xi^{\prime} \cos \phi^{\prime}+K_{y} \frac{\beta^{\prime}}{\alpha^{\prime}} \xi^{\prime} \sin \phi^{\prime}+K_{z} \eta^{\prime}\right) d \phi d \phi^{\prime} d \eta d \eta^{\prime} .
\end{aligned}
$$

It is convenient now to introduce the transformation

$K_{x}=(1 / \alpha) r \cos \omega \sin \psi, \quad K_{y}=(1 / \alpha) r \sin \omega \sin \psi, \quad K_{z}=(1 / \xi) r \cos \psi$, with

$$
0 \leqq r \leqq \infty, \quad 0 \leqq \psi \leqq \pi, \quad 0 \leqq \omega<2 \pi .
$$

When this is done, the $\phi$ and $\phi^{\prime}$ integrations, which are separable, take on known forms which yield Bessel functions, and the $\eta$ and $\eta^{\prime}$ integrals are also separables and are covered by the form (14). Upon performing these integrations, the $\omega$ dependence of the integrand disappears, and the above expression may be written

$$
\begin{aligned}
C_{\mu \nu}=- & \frac{16 \pi^{2} i^{\mu+\nu} F^{3} \alpha^{4} \xi}{(2 \mu+1)(2 \nu+1)} \lim _{\xi^{\prime} \rightarrow \xi} \int_{0}^{\pi} \frac{\sin \psi d \psi}{\xi^{2}-\cos ^{2} \psi} \int_{0}^{\infty} \frac{r^{3} d r}{r^{2}-c^{2}} \\
& \cdot\left\{\frac{\xi \sin \psi}{\alpha^{2}}\left[P_{\mu-1}^{1}(\cos \psi) J_{\mu-\frac{1}{2}}(F r)+P_{\mu+1}^{1}(\cos \psi) J_{\mu+\frac{3}{2}}(F r)\right]\right. \\
- & \left.\frac{\cos \psi}{\xi}\left[(\mu+1) P_{\mu+1}(\cos \psi) J_{\mu+\frac{3}{2}}(F r)-\mu P_{\mu-1}(\cos \psi) J_{\mu-\frac{1}{2}}(F r)\right]\right\} \\
& \cdot\left\{\frac{\xi^{\prime} \sin \psi}{\alpha \alpha^{\prime}}\left[P_{\nu-1}^{1}(\cos \sigma) J_{\nu-\frac{1}{2}}(F r \epsilon)+P_{\nu+1}^{1}(\cos \sigma) J_{\nu+\frac{3}{2}}(F r \epsilon)\right]\right. \\
+ & \left.\frac{\cos \psi}{\xi}\left[(\nu+1) P_{\nu+1}(\cos \sigma) J_{\nu+\frac{3}{2}}(F r \epsilon)-\nu P_{\nu-1}(\cos \sigma) J_{\nu-\frac{1}{2}}(F r \epsilon)\right]\right\}
\end{aligned}
$$

where

$$
\begin{gathered}
-\left(\xi^{\prime} / \xi\right) \cos \psi \equiv \epsilon \cos \sigma, \quad\left(\alpha^{\prime} / \alpha\right) \sin \psi \equiv \epsilon \sin \sigma, \quad \text { and } \\
c^{2} \equiv k^{2} \xi^{2} \alpha^{2} /\left(\xi^{2}-\cos ^{2} \psi\right) .
\end{gathered}
$$

It can be seen from the above form that the integral vanishes when $\mu+\nu$ is odd. The $r$ integrations now take on the form

$$
R_{m n} \equiv \int_{0}^{\infty} J_{m+\frac{1}{2}}(F r) J_{n+\frac{1}{2}}(F r \epsilon) \frac{r^{3} d r}{r^{2}-c^{2}}
$$

with $m+n$ even. The integrand here is then an even function about $r=0$ which oscillates indefinitely with constant amplitude as $r \rightarrow \pm \infty$ so that the integral itself is not strictly convergent. However, a preliminary integration 
over $\epsilon$ yields a function which is integrable in $r$ and the derivative of the integral with respect to $\epsilon$ represents a formal value of the $r$ integral. The actual $r$ integration is carried out by means of a contour integral. Writing $J_{m+\frac{1}{2}}(F r)$ as the sum of Hankel functions of the first and second kinds, we have two functions, both even about $r=0$, but one of which vanishes as $r \rightarrow+i \infty$ and the other as $r \rightarrow-i \infty$. The contours then consist of the real axis from $-\infty$ to $+\infty$ but with deviations above the point $r=-c$ and below $r=+c$, as specified by the representation used for the Green's function, closed by infinite semicircles in the upper half-plane for the term containing $H_{m+\frac{1}{1}}^{(1)}$, or in the lower for the term containing $H_{m+\frac{1}{2}}^{(2)}$. The resulting form for the $r$ integrals is

$$
R_{m n}=\frac{1}{2} \pi i c^{2} \mathrm{H}_{m+\frac{1}{3}}^{(1)}(F c) J_{n+\frac{1}{3}}(F c \epsilon) .
$$

The limit as $\xi^{\prime} \rightarrow \xi$ can now be taken, and the resulting $\psi$-integrand in equation (16) factored to give the form (9).

Acknowledgments. The author wishes to express deep appreciation to his thesis advisor, Professor Wilhelm Magnus of New York University, for prompt and invaluable aid and advice; to his colleagues, in particular K. M. Siegel, C. E. Schensted, and A. H. Halpin, for illuminating discussions of the problem; and to H. E. Hunter for his meticulous work in computing the numerical results.

Added in proof. The fairly rapid convergence of the variational solution for moderate values of $k a$ indicated above is not surprising in view of the analysis of Jones." As applied here the variational form can be considered an "exact" solution in the usual sense of the word. This is perhaps more strikingly brought out by the following observation.

If the trial function $\phi\left(s^{\prime}\right)$ of equation (3) is expanded in spheroidal functions instead of spherical ones and if a corresponding expansion of the Green's function in spheroidal functions is used in place of the Fourier integral form employed above, then the variational solution reduces almost immediately to a form which is identical to that yielded by the wave-function solution. The forms developed here thus would seem to contain implicitly the spheroidal coefficients which relate the spheroidal functions to the spherical ones. The explicit expression of these coefficients by means of these forms, however, appears to present some difficulty.

The University of Michigan

RADIATtON LABORATORY

(Received November 5, 1958) 